\title{
Loss of ciliary activity in organ cultures of rat trachea treated with lipo-oligosaccharide from Haemophilus influenzae
}

\author{
A. P. JOHNSON and T. J. INZANA* \\ Division of Sexually Transmitted Diseases, MRC Clinical Research Centre, Harrow, Middlesex, HA1 3UJ, and \\ *Department of Veterinary Microbiology-Pathology, College of Veterinary Medicine, Washington State \\ University, WA 99164-7040, USA
}

\begin{abstract}
Summary. Organ culture of rat trachea was used as an experimental model to examine the ability of lipo-oligosaccharide from Haemophilus influenzae to damage respiratory tract mucosal tissue. Lipo-oligosaccharide from two strains of $H$. influenzae produced a significant decrease in the ciliary activity of tracheal rings observed over a 3-5 day period. No loss of ciliary activity was observed with the lipid-free moiety of the lipooligosaccharide.
\end{abstract}

\section{Introduction}

Haemophilus influenzae is the commonest cause of bacterial meningitis in infants and young children and may also cause other serious systemic infections in this age group. It is also associated with exacerbations of chronic bronchitis in adults (Turk, 1982). Despite the importance of $H$. influenzae as a human pathogen, its pathogenicity is incompletely understood. To increase our understanding of this, several experimental systems, including an animal model of meningitis (Moxon et al., 1974) and the use of organ cultures of respiratory mucosa inoculated with $H$. influenzae (Denny, 1974; Johnson et al., 1983) have been developed. With the latter system it was shown that ciliary activity in cultures of tracheal mucosa inoculated with $H$. influenzae was lost because of sloughing of epithelial cells (Denny, 1974; Johnson et al., 1983). It was also observed that sterile supernates from infected organ cultures contained a soluble, heat-stable factor that caused loss of ciliary activity when transferred to fresh organ cultures (Denny, 1974). Although the heat-stable toxic factor was not identified, Denny speculated that it might be $H$. influenzae endotoxin. In the present study, the concept that $H$. influenzae endotoxin might be capable of damaging tracheal mucosa has been investigated by determining the effect that purified lipo-oligosaccharide from $H$. influenzae has on the ciliary activity of organ cultures of rat trachea.

Received 12 Mar. 1986; accepted 19 Mar. 1986

\section{Materials and methods}

\section{Lipo-oligosaccharide}

Lipo-oligosaccharide (LOS) was extracted from $H$. influenzae strains 1060 and S-2. Strain 1060 was isolated from a child with meningitis at the Texas Children's Hospital, Houston, TX. Strain S-2 is a capsule-deficient mutant of strain Eagan (Moxon et al., 1984). The LOS was isolated from each strain by enzyme digestion and phenol-water extraction (Johnson and Perry, 1976; Inzana, 1983). Lipid-free oligosaccharide was isolated from strain Eagan as described by Inzana et al. (1985). The samples of LOS and oligosaccharide were prepared in the laboratory of one of the authors (TJI) and after lyophilisation, were sent to APJ for assessment of their biological activity in rat trachea organ cultures.

\section{Preparation of organ cultures of rat trahcea}

Young male and female specific-pathogen-free Sprague-Dawley or Lewis rats, bred at the Clinical Research Centre, were used. Tracheas were removed aseptically from animals by the method of Johnson et al. (1983) and placed in a petri-dish containing Eagle's minimal essential medium (MEM) buffered at $p \mathrm{H} 7.2$ with 50 mM HEPES and supplemented with ampicillin $10 \mu \mathrm{g} / \mathrm{ml}$. The MEM was prepared with sterile pyrogen-free water as described by Johnson et al. (1983). Each trachea was cut into a series of rings which were placed individually in tissue-culture tubes containing $1 \mathrm{ml}$ of MEM and then incubated at $37^{\circ} \mathrm{C}$ on a roller drum (Cherry and Taylor-Robinson, 1970).

\section{Treatment of organ cultures}

In initial experiments, organ cultures were incubated 
on a roller drum at $37^{\circ} \mathrm{C}$ overnight and inspected to determine whether or not the ciliated mucosal cells were still active (see below). The medium was then removed and replaced with $1 \mathrm{ml}$ of MEM supplemented with ampicillin and containing LOS or lipid-free oligosaccharide. Control organ cultures were maintained in MEM only. The ciliary activity of each ring was then assessed at daily intervals. Later experiments were performed as described above, but with the modification that organ cultures were not incubated overnight before treatment with LOS.

\section{Ciliary activity}

The percentage of the mucosal surface of each tracheal ring with actively beating cilia was determined by visual observation with an inverted microscope as described by Cherry and Taylor-Robinson (1970). To increase the objectivity of the visual assessment of ciliary activity, the organ cultures were coded before examination and the observer did not know which rings belonged to the treatment and control groups.

\section{Experimental design and statistical analysis}

With the exception of a preliminary experiment in which tracheal rings from only one animal were used, each experiment was performed with tracheal rings from two animals of the same age and strain. Half of the rings from each trachea were treated with LOS or lipid-free oligosaccharide and the remaining rings were maintained in medium as controls. The significance of the difference of ciliary activity between treated and untreated control organ cultures was determined by Wilcoxon's Sum of Ranks Test (two-sample test for unpaired data) (Swinscow, 1976).

\section{Results}

\section{Effect of LOS on ciliary activity}

In a preliminary experiment with LOS from strain 1060, organ cultures of trachea from Sprague-Dawley rats were treated with LOS at concentrations of either $25 \mu \mathrm{g} / \mathrm{ml}$ or $2.5 \mu \mathrm{g} / \mathrm{ml}$. By 3 days

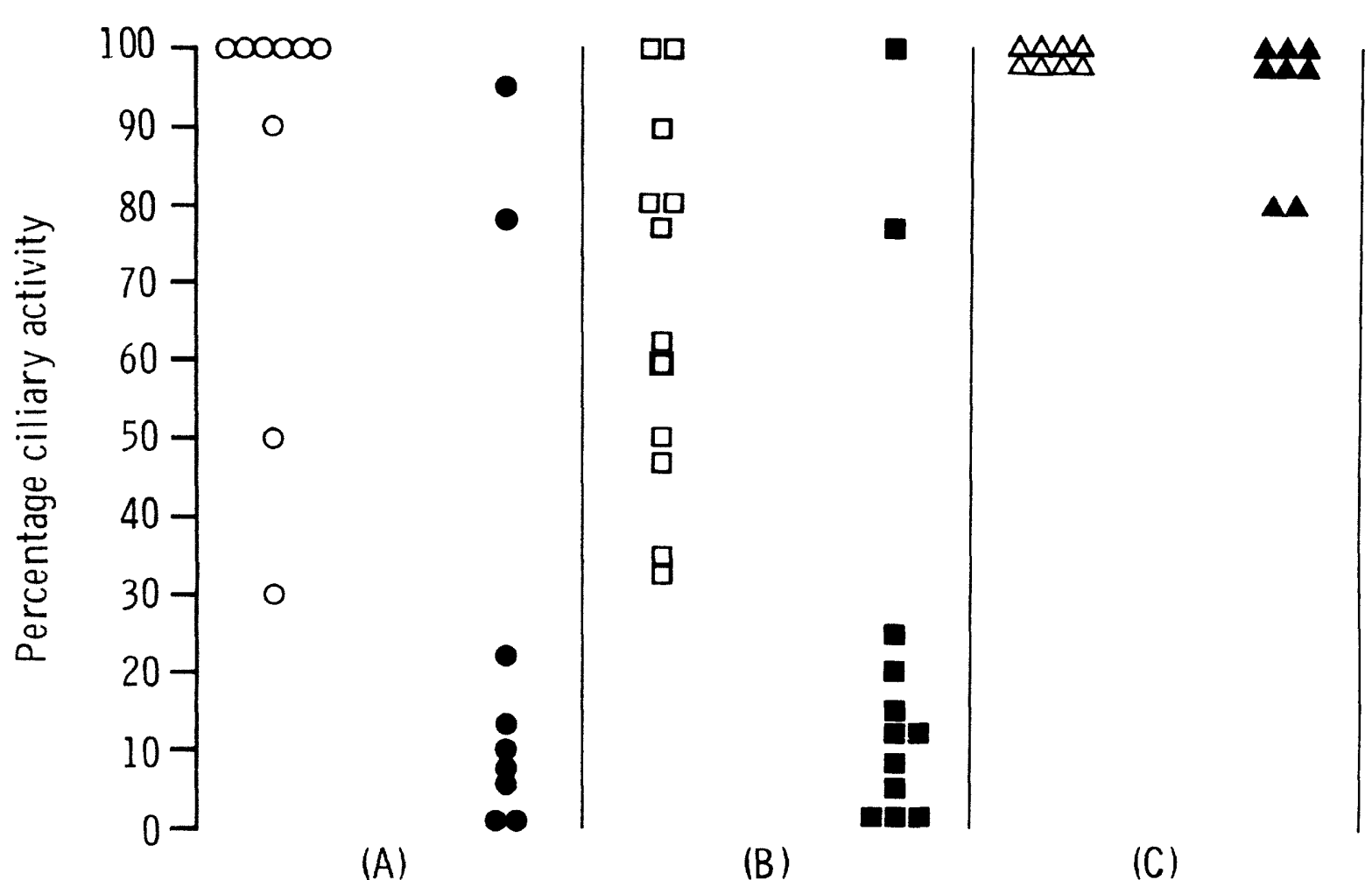

Fig. Ciliary activity of organ cultures of rat trachea maintained either in MEM alone $(O, \square, \triangle)$ or in MEM supplemented with LOS $(\bullet)$ or oligosaccharide ( $\triangle$ ). (A) Ciliary activity after observation for 4 days; LOS from strain 1060 was used at a concentration of 25 $\mu \mathrm{g} / \mathrm{ml}$. (B) Ciliary activity after observation for 5 days; LOS from strain S-2 was used at a concentration of $40 \mu \mathrm{g} / \mathrm{ml}$. (C) ciliary activity after observation for 5 days; oligosaccharide from strain Eagan was used at a concentration of $40 \mu \mathrm{g} / \mathrm{ml}$. 
after inoculation, each of three tracheal rings treated with $25 \mu \mathrm{g} / \mathrm{ml}$ showed $\geqslant 50 \%$ loss of ciliary activity, while each of three untreated organ cultures and two of three organ cultures treated with $2.5 \mu \mathrm{g} / \mathrm{ml}$ showed $\leqslant 10 \%$ decrease in ciliary beating. Therefore in further studies with LOS from strain 1060 LOS was used at a concentration of $25 \mu \mathrm{g} / \mathrm{ml}$. As shown in the figure (Aa), organ cultures of trachea from Sprague-Dawley rats treated with LOS showed a statistically significant decrease in ciliary activity when compared to untreated organ cultures after observation for 4 days $(p=0.016)$. After these experiments, the unavailability of Sprague-Dawley rats meant that subsequent studies had to be performed with tracheas from Lewis rats. In one experiment, tracheal rings from a Lewis rat were found to be susceptible to damage by LOS from $H$. influenzae strain 1060 ; there was $>60 \%$ decrease in ciliary activity after observation for 4 days (data not shown). Thus it appeared that tracheal tissue from both Sprague-Dawley and Lewis rats was susceptible to the toxic effect of LOS.

A second group of experiments was then performed in which the effect of LOS fom $H$. influenzae strain S-2 on the ciliary activity of tracheal rings was assessed. In these studies, the concentration of LOS was increased to $40 \mu \mathrm{g} / \mathrm{ml}$ in an attempt to shorten the length of time required for loss of ciliary activity to be observed. As in the experiments described above, organ cultures of rat trachea treated with LOS from strain S-2 showed a statistically significant decrease in ciliary activity in comparison to untreated control cultures $(p=0.002)$ (figure, B). However, despite the use of a higher concentration of LOS, the time for loss of ciliary activity to become apparent (3-5 days) was similar to that seen in experiments with LOS from strain 1060.

\section{Effect of lipid-free oligosaccharide on ciliary activity}

One experiment was performed to test the effect of the oligosaccharide fraction of LOS from strain Eagan on ciliary beating. As shown in the figure (C), oligosaccharide at a concentration of $40 \mu \mathrm{g} / \mathrm{ml}$ had no significant effect on the ciliary activity of tracheal organ cultures up to 5 days after treatment. Six of eight tracheal rings treated with oligosaccharide showed no loss of ciliary beating and the other two rings retained $80 \%$ of their original activity.

\section{Discussion}

The results presented here show that LOS samples from two strains of $H$. influenzae can produce mucosal damage, manifest as loss of ciliary activity, in organ cultures of rat trachea. These findings are consistent with the observation that $H$. influenzae produces a heat-labile, non-dialysable product that inhibits ciliary beating in cultures of rat trachea (Denny, 1974). Interestingly, another group of investigators, working with organ cultures of ciliated human nasal epithelium (Wilson et al., 1985) have also recently observed that LOS from $H$. influenzae induces sloughing of epithelial cells ( $R$. Wilson, personal communication).

These findings are consistent with the observation that LOS from Neisseria gonorrhoeae produces loss of ciliary activity in organ cultures of fallopian tube (Gregg et al., 1981). The fact that LOS preparations from two different genera of bacteria are both toxic to ciliated mucosal surfaces suggests that there may be value in assessing the ciliostatic potential of LOS from other bacteria which colonise and damage mucosal surfaces, particularly those of the respiratory and upper genital tracts.

The mechanism by which LOS produces mucosal damage is not known, although the finding that gonococcal LOS is toxic for human but not nonhuman genital tissue (Gregg et et., 1981) implies specific interaction between LOS and the epithelial surface. The finding that rat trachea is susceptible to the toxic effect of LOS from $H$. influenzae is not surprising because rats are known to be susceptible to infection with this organism (Moxon et al., 1974). Studies with gonococcal LOS have indicated that the lipid A portion of the LOS molecule is involved in producing tissue damage (Gregg et al., 1981). The present finding that the oligosaccharide moiety of LOS from $H$. influenzae is non-toxic adds further weight to the idea that lipid $A$ is the active component, although it would be premature to state that the oligosaccharide portion of LOS has no role in the production of tissue damage.

If the ability of LOS from $H$. influenzae to damage ciliated mucosal tissue in vitro is also manifest in vivo it could constitute an important virulence factor. The mucociliary lining covering the mucosa of much of the respiratory tract provides an important non-specific host defence mechanism, by mechanically inhibiting micro-organisms from readily colonising this site. If LOS acted to reduce mucociliary clearance of organisms by the host it would enhance their ability to colonise and infect the respiratory tract. The sloughing of epithelial cells from the surface of the respiratory tract, which has been observed in vitro (Johnson et al., 1983) might also provide a means by which $H$. influenzae can cross the respiratory epithelium to invade the submucosal tissue, with subsequent systemic spread. 


\section{REFERENCES}

Cherry J D, Taylor-Robinson D 1970 Large-quantity production of chicken embryo tracheal organ cultures and their use in virus and mycoplasma studies. Applied Microbiology 19:658-662.

Denny F W 1974 Effect of a toxin produced by Haemophilus influenzae on ciliated respiratory epithelium. Journal of Infectious Diseases 129:93-100.

Gregg C R, Melly M A, Hellerqvist C G, Coniglio J G, McGee Z A 1981 Toxic activity of purified lipopolysaccharide of Neisseria gonorrhoeae for human fallopian tube mucosa. Journal of Infectious Diseases 143:432-439.

Inzana T J 1983 Electrophoretic heterogeneity and interstrain variation of the lipopolysaccharide of Haemophilus influenzae. Journal of Infectious Diseases 148:492-499.

Inzana T J, Seifert W E, Williams R P 1985 Composition and antigenic activity of the oligosaccharide moiety of Haemo- philus influenzae type $\mathrm{b}$ lipo-oligosaccharide. Infection and Immunity 48:324-330.

Johnson A P, Clark J B, Osborn M F 1983 Scanning electron microscopy of the interaction between Haemophilus influenzae and organ cultures of rat trachea. Journal of Medical Microbiology 16:477-482.

Johnson K G, Perry M B, 1976 Improved techniques for the preparation of bacterial lipopolysaccharides. Canadian Journal of Microbiology 22:29-34.

Moxon E R, Smith, A L, Averill D R, Smith D H 1974 Haemophilus influenzae meningitis in infant rats after intranasal inoculation. Journal of Infectious Diseases 129:154-162.

Swinscow T D V 1976 Statistics at square one. XVII-some nonparametric tests. British Medical Journal 2:632-634.

Turk D C 1982 Haemophilus influenzae. Public Health Laboratory Service Monograph series No. 17, HMSO, London.

Wilson R, Roberts D, Cole P 1985 Effect of bacterial products on human ciliary function in vitro. Thorax 40:125-131. 